\title{
THE COMPLICATIONS OF RESERPINE THERAPY
}

\author{
By J. G. Macarthur, M.C., M.B., M.R.C.P. \\ Senior Lecturer in Materia Medica and Therapeutics, University of Glasgow
}

Preparations of Rauwolfia have been used in Indian folk medicine for centuries in the treatment of a great variety of diseases. The actions and uses of extracts of Rauwolfia root have been studied by Indian pharmacologists and clinicians for over 25 years but it was not until Vakil (1949) drew attention to the value of the drug in the treatment of arterial hypertension that interest was aroused in the Western world. In 1952, Müller, Schlittler and Bein isolated the pure alkaloid reserpine from Rauwolfia serpentina Benth., and since then the drug has been used in the treatment of arterial hypertension and in psychiatric disorders throughout the entire world. Its usefulness in these conditions has been established but at the same time many side effects of therapy, few of which fortunately are serious, have been noted. This paper gives an account of the complications of reserpine therapy.

Reserpine, acting centrally on both somatic and autonomic nervous pathways, exhibits a complex pattern of activity. Characteristic features include sedation of a peculiar type, lowering of arterial blood pressure accompanied by bradycardia, vasodilatation, stimulation of peristalsis and some respiratory inhibition. It is not therefore surprising that some side effects are inevitable accompaniments when the drug is used therapeutically to lower blood pressure or to produce a sedative effect in psychiatric practice.

Three preparations are in common use; a whole root preparation (' Raudixin'), a mixed alkaloid preparation free from inactive material ('Rauwiloid ') and the pure alkaloid reserpine. No significant differences in the side effects produced have been found with these preparations, but when large doses have been given to psychotic patients the pure alkaloid has usually been used. In the treatment of arterial hypertension doses of the order of $\mathrm{I} \mathrm{mg}$. daily of reserpine or equivalent dosage of the cruder preparations have commonly been employed but in the treatment of psychiatric cases such doses have usually been found to be ineffective, at least in the early stages of therapy, and much larger doses are often used. Reserpine has been given both orally and parenterally in amounts as great as $60 \mathrm{mg}$. or more daily. The $\overrightarrow{\vec{\omega}}$ incidence and severity of most of the side effects $\stackrel{\omega}{\sigma}$ increases with increased dosage but psychotic $\overrightarrow{8}$ patients tolerate such large doses remarkably well. 3 No doubt the hypertensive patient is more likely to complain of annoying side effects than patients $i \omega$ with mental illness and this may explain in part at of least the tolerance displayed by the latter group despite the much higher dosage of the drug.

Unfortunately, the exact sites of action of $\frac{\text { O }}{7}$ reserpine and its mode of action at cellular level have not been fully defined, and it is therefore not possible to give a pharmacological explanation for many of the complications seen in clinical practice and described below.

The sources, chemistry and pharmacologic actions of the Rauwolfia alkaloids have recentl been reviewed by Lewis (1956) and by Bein (1956) and the reader is referred to these admirable accounts for further details of present knowledge on the actions of Rauwolfia. A full list of references is given by each author.

\section{Fatalities}

Reserpine has a very high therapeutic index. It has been estimated that the lethal dose in man is around $280 \mathrm{mg}$. given intravenously. A child of 20 months has survived after accidental ingestion of $200 \mathrm{mg}$. of the drug. Accordingly, fatalities following its therapeutic use are very rare. Death has followed electroconvulsant therapy in patients receiving large doses of reserpine, and non-fatal severe reactions with prolonged hypotension and respiratory inhibition have also been noted with such combined therapy. Fatal reactions have also occurred when large doses of the drug have been given by the intravenous route to acutely agitated psychotic patients and in alcoholic delirium tremens. A fatal outcome has also been seen in in status epilepticus apparently induced by reserpine. Death results from centrally conditioned respiratory failure.

\section{Nervous System}

The main action of reserpine is exerted on the central nervous system leading to sedation. The 
type of action however can be differentiated from that of other well-known drugs with a central depressant action. Reserpine facilitates sleep but does not induce it and the patient under its influence is easily aroused. The majority of patients experience some degree of lethargy, sedation and inability to concentrate. In agitated psychotic patients these effects are desirable but when the drug is used to lower the arterial blood pressure many patients complain of these effects. Excessive dreaming and nightmares are also not infrequent. During the early phases of treatment, especially with large doses, irritability, mild confusion, nervousness, agitation and apprehension may appear and as treatment is continued these may give way to sedation in mentally disturbed patients. When such symptoms appear however in hypertensive subjects under treatment with small doses of the drug, they are often a prelude to serious organic depression and indicate that great care be used in continuing therapy. The warning signs are lethargy and drowsiness by day with agitation, apprehension and insomnia by night. Such patients complain of a feeling of isolation or strangeness and such statements as 'I don't feel like myself' are common. If reserpine treatment is discontinued at this stage in patients without a previous history of depression, recovery follows. There is some evidence that phenidylate ('Ritalin'), a new anti-depressant drug, may prevent the occurrence of minor depressive states but further study is needed before final conclusions can be drawn. In severe reserpine-induced depression it is ineffective. Burrell (1956) claims that sodium succinate is of value in the treatment of reserpineinduced depression. This observation is of interest as Gillis and Lewis (1956) working in this department have shown that certain intermediates of the tricarboxylic acid cycle antagonise the action of reserpine on isolated guinea pig ileum.

\section{Mental Depression}

The most serious complication of reserpine therapy in hypertensive subjects is the occurrence of reactive depression indistinguishable from ordinary depressive illness. It does not disappear when the drug is withdrawn and about half of the patients require active psychiatric treatment including ECT to effect recovery. Several instances of suicide have occurred during the druginduced depressive illness. The incidence varies from 5 to 20 per cent. of patients in different series and it is less the smaller the dose of reserpine employed. The large majority of the cases has occurred in hypertensive patients treated with relatively small doses of the drug and it has not been reported frequently when large doses are used in the treatment of psychotic patients. However, Faucett it al. (1957) have recently claimed that agitated depression becomes much worse with reserpine therapy and that such subjects are more likely to develop a Parkinsonian syndrome (see below). A previous history of depression makes this complication much more likely but it has often occurred when careful psychiatric analysis has failed to show any previous history of mental disturbance. Reserpine seems to precipitate the abnormal response in these cases, though the majority of individuals will not develop a depressive illness even when large doses of the drug are given. Faucett and his colleagues think that reserpine acts in and around the diffuse thalamic projection system and paleocortex, that the patient seeks the cause of his altered state in his external environment and reacts with a depressive illness. When the drug is withdrawn the psychological misinterpretation remains until therapy corrects it.

The prevention of this serious drawback to reserpine treatment of arterial hypertension lies in the use of the smallest effective dose, preferably not more than $0.5 \mathrm{mg}$. daily, and early recognition of the warning signs with discontinuation of treatment. Reserpine should be used with greato caution, if at all, in patients with a history of de-0 pression. Smirk (1956) has stated that rescinna mine and canescine are less likely to produce mental depression than reserpine.

\section{Parkinsonism}

This unusual response to drug therapy was known to the early Indian workers (Sen and Bose, I93I) and is of particular interest in relation to the site of action of reserpine. Unlike the depressive states described above, the Parkinson-like syndrome seems to be a function of the dose employed. A progressive rise in the incidence of the syndrome is seen with increase in dosage and duration of treatment. At dosage levels of over $30 \mathrm{mg}$. daily employed for more than two months the incidence is very high. It is more easily produced in younger subjects than in the elderly, and as noted above may be particularly liable to occur in depressed patients treated with large doses of the drug. The features include Parkinsonian facies, muscular rigidity of extra pyramidal type, slowness of movement, coarse tremor, shuffling gait and salivation. The symptoms are reversible and disappear, sometimes slowly, when the drug is withdrawn. It is thus truly a feature of overdosage and has also been noted in patients treated with chlorpromazine. The site of action of reserpine in the production of the syndrome has not been elucidated. 


\section{Miscellaneous Central Nervous System Effects}

Early in the course of treatment with reserpine epileptiform convulsions may be observed in patients for the first time and in epilepsy the convulsions may increase in frequency. This is in accord with the finding in experimental animals that reserpine lowers the convulsant threshold. Occasionally however lessening of the frequency of seizures has been reported when reserpine in moderate doses has been combined with anticonvulsant therapy. Reference has already been made to the necessity for using ECT with care in patients being treated with reserpine. Severe generalised chorea has also been noted, but on the other hand improvement has occurred in some cases of Huntingdon's chorea treated with reserpine. Acute cerebellar ataxia has also been described with rapid recovery when the drug was discontinued. Of exceptional occurrence is acute delirium and unrousable coma as manifestations of overdosage.

Despite intensive study the exact sites of action of reserpine upon the brain have not yet been elucidated and it is not yet possible to give a clear account of the pathways involved in the production of these effects. The mode of action at cellular level has not been defined and the possible interaction between reserpine or its metabolites and 5-hydroxytryptamine in producing the actions in the central nervous system must await further neuro-pharmacological research.

\section{Cardiovascular System Side Effects}

Reserpine has been generally held to lower arterial blood pressure by causing vasodilatation of central origin due to sympathetic outflow inhibition at hypothalamic levels, but in addition there is probably a peripheral component of this action (Lewis, 1957). Postural hypotension is uncommon and does not usually constitute a problem even when very large doses are given. Nasal congestion, conjunctival suffusion and flushing of the skin over the blush areas is often seen and many patients complain of the nasal stuffiness induced by the congested mucosa. Nasal congestion also occurs in the new born infants of mothers treated with large doses of reserpine for pregnancy toxaemia. The nasal congestion is relieved rather inefficiently by antihistaminic drugs and only occasionally by sympathomimetic vasoconstrictors. These facts have led to the suggestion that the flushing and nasal congestion may be contributed to, at least in part, by increased amounts of circulating 5-hydroxytryptamine, which is known to be released from the cells of the intestine, brain, spleen and platelets by reserpine. There is no proof, however, that this mechanism plays any part in the production of these effects.

\section{Cardiac Effects}

The bradycardia which accompanies the fall in arterial blood pressure does not give rise to symptoms. A few reports have appeared of the occurrence of premature ventricular contractions in patients being treated with reserpine and they are apparently more likely to appear in the digitalized patient. Paroxysmal rhythm change has not been encountered and the effect is uncommon and unimportant. The mechanism is unknown.

\section{Salt and Water Retention and Heart Failure}

Oedema of the face and extremities is sometimes noticed in patients treated with reserpine, especially when large doses are used. In the presence of severe hypertensive heart disease the salt and water retention may cause sufficient circulatory overload to precipitate frank heart failure. In the former group withdrawal of the drug leads to diuresis and disappearance of the oedema. Indeed in some patients the oedema may disappear even when therapy is continued. In the patients with severe hypertensive heart disease, measures to control heart failure ando promote diuresis may be needed as well as dis continuation of reserpine treatment. The mecheo anism of production of salt and water retention is? unknown. Consistent changes in renal haemodynamics have not been found in patients treated with reserpine; increased output of adrenocortical steroid has been shown to occur in experimental animals given large doses of reserpine. Hulet and Perera (1956) have also shown that 5-hydroxytryptamine infused intravenously induces sodium retention in human subjects. The elucidation of the problem must await further research.

\section{Alimentary Side Effects}

Reference has already been made to the salivation which accompanies the Parkinsonian syndrome induced by reserpine and this effect is sometimes seen when large doses are given in the absence of Parkinsonism. It can be controlled with atropine and other anticholinergic drugs.

Occasionally nausea and vomiting occur in patients given large doses of the drug and these effects have been thought to be due to increased gastric motility, increased output of hydrochloric acid and possibly vasodilation of the vessels of the gastric mucosa. A much more important side effect is the occasional occurrence of gastroduodenal haemorrhage even in patients who give no previous history of peptic ulceration. The 
haemorrhage has been presumed in such cases to be due to gastric erosion occasioned by the abovementioned hypermotility and hypersecretion accompanied by mucosal vasodilation. In persons with gastro-duodenal ulceration it is obviously desirable that reserpine be used with care, especially if large doses are contemplated. Diarrhoea is a common symptom at all levels of reserpine dosage and is due to increased gastrointestinal activity. This effect is antagonized by anticholinergic drugs and by ganglion blocking agents. Indeed in hypertensive subjects treated with a combination of reserpine and ganglion blocking agent the constipation often produced by the latter remedy is relieved by the concomitant reserpine therapy. A full explanation of the mode of action of reserpine on the alimentary tract is not yet available.

\section{Respiratory Side Effects}

It has already been noted that death from reserpine poisoning is due to central respiratory failure and reference has also been made to the respiratory distress which may occur when electroshock therapy is given to patients treated with large doses of the drug. Reserpine may also on occasion induce bronchospasm in susceptible individuals and exertional dyspnoea may occur when the drug produces salt and water retention.

\section{Endocrine Side Effects}

Increased appetite with weight gain is of common occurrence during reserpine therapy. It may be especially marked in mentally ill patients, who have lost weight during their illness. In such patients the increase in appetite may amount to polyphagia. This effect of reserpine has not been analyzed in detail.

Reference has already been made to the occurrence of water and salt retention and the possible endocrine mechanisms involved. It may be especially marked in women during the premenstrual phase. In male patients, but apparently not in women, there may be a decrease in libido, and gynaecomastia has also been reported in the male. The production of lactation in nonpregnant women is an occasional occurrence and inhibition of lactation has been produced in the immediate post-partum period. No explanation of these effects can yet be offered.

\section{Miscellaneous Side Effects}

Sundry inexplicable side effects have often been reported in the literature. These include vague feelings of chilliness with aching in the limbs in the absence of demonstrable changes in body temperature. Indefinite malaise and 'limb weariness' are also not infrequent. These symptoms tend to disappear with continued therapy. In psychotic patients reserpine may modify the clinical picture of intercurrent physical illness so that pain, fever and leucocytosis may be minimal. Headache and dizziness are often listed among the side effects but these symptoms are so common in hypertensive and mentally disturbed patients that their relation to treatment with reserpine is difficult to estimate.

\section{Idiosyncrasy}

This seems to be very rare. One case of severe angioneurotic oedema has been reported and occasionally skin rashes have been noted. No instance of hepatic damage nor of depression of the bone marrow has been reported even after prolonged therapy with large doses of the drug.

\section{Overall Incidence of Side Effects}

The incidence of side effects varies greatly in different publishéd series of cases treated with reserpine, and it is greater the higher the daily dose of the drug employed. In general it can be said that some degree of sedation, nasal congestion and increase in appetite is experienced by the great majority of patients. Nearly one half of patients treated experience excessive dreaming and in about one quarter a laxative effect is noted. Twenty per cent. of hypertensive patients experience some degree of mental depression, but this effect can be minimized by reduction of the daily dose to not more than $0.5 \mathrm{mg}$. daily. Further experience with rescinnamine and canescine may indicate that these alkaloids are less likely to provoke this serious complication. Parkinsonism can be produced regularly if large doses of reserpine are given over a long period of time. The other side effects appear in less than ro per cent. of cases treated and some, such as gastroduodenal haemorrhage, are of exceptional occurrence. The drug is remarkably free from sensitivity reactions and liver damage and blood dyscrasia following its use have not been reported.

In conclusion, it can be said that while the main indications for reserpine therapy and the complications arising therefrom have been reasonably defined, the many problems related to its mode of action await solution.

\section{BIBLIOGRAPHY}

BEIN, H. J. (1956), Pharmacol. Rev., 8, 435.

BURRELL, R. H.' (1956), N.Z. med. $尹$., $55,228$.

FAUCETT, R. L., LITIN, E. M., and ACHOR, W. P. (1957), A.M.A.' Arch. Neurol. Psychiat., 77, 513 .

GILLIS, C. N., and LEWIS, J. J. (1956), Nature, 178, 859

HULET, W. H., and PERERA, G. A. (1956), Proc. Soc. exp. Biol.,

LEWI, 512. J. (1956), 7. Pharm. Pharmacol., 8, 465.

MULLER. J. M., SCHLITTLER, E., and BEIN, H. J. (1952), MULLER, J. M., SCHLITTL

SEN, G., and BOSE, K. (I93I), Indian med. World, 2, 194 SMIRK, F. H. (1956), 'Symposium on Hypotensive Drugs', Pergamon Press, London and New York, p. 113. VAKIL, R. J. (1949), Brit. Heart $\mathcal{Y}$., II, 350 . 